\title{
Importance of nutritional therapy in the management of intestinal diseases: beyond energy and nutrient supply
}

\author{
Seong-Eun Kim \\ Department of Internal Medicine, Ewha Womans University College of Medicine, Seoul, Korea
}

The gut is an immune-microbiome-epithelial complex. Gut microbiome-host interactions have widespread biological implications, and the role of this complex system extends beyond the digestion of food and nutrient absorption. Dietary nutrients can affect this complex and play a key role in determining gut homeostasis to maintain host health. In this article, we review various dietary nutrients and their contribution to the pathogenesis and treatment of various intestinal diseases including inflammatory bowel disease, irritable bowel syndrome, colorectal cancer, and diverticulitis, among other such disorders. A better understanding of diet-host-gut microbiome interactions is essential to provide beneficial nutrients for gut health and to limit nutritional hazards to ensure successful nutritional management of gastrointestinal conditions in clinical practice. (Intest Res 2019;17:443-454)

Key Words: Nutrition therapy; Gut microbiome; Diet

\section{INTRODUCTION}

The rapid pace of development and globalization has contributed to significant and widespread changes in lifestyle and dietary habits in society. Previously, nutritional management of intestinal diseases was primarily aimed at ensuring an adequate supply of energy and protein to ensure recovery from malnutrition secondary to GI disorders. However, the recent concept of nutritional therapy has been further expanded. Multiple lines of evidence suggest that diet plays a role in the onset, perpetuation, and management of various intestinal diseases. With regard to pathogenesis, several dietary nutrients have been shown to affect the host immune system and the intestinal barrier function, as well as the composition and function of gut microbiome. ${ }^{1-5}$

We review the current knowledge regarding the role of di-

Received June 10, 2019. Revised July 1, 2019. Accepted July 5, 2019 Correspondence to Seong-Eun Kim, Department of Internal Medicine, Ewha Womans University College of Medicine, 1071 Anyangcheon-ro, Yangcheongu, Seoul 07985, Korea. Tel: +82-2-2650-2845, Fax: +82-50-4272-6509,

E-mail: kimse@ewha.ac.kr etary nutrients in the pathogenesis and treatment of various intestinal diseases. We have specifically discussed research findings describing the interplay between dietary nutrients, the gut microbiome, and the host immune and gut barrier systems. A deeper understanding of the gut-host-microbiome interactions may aid in the development of a reliable preventive strategy for intestinal diseases, as well as promote newer therapeutic approaches for several diseases encountered in clinical practice.

\section{THE GUT MICROBIOME-DIET-IMMUNE COMPLEX: BEYOND A NUTRIENT ABSORPTION ORGAN}

The gut primarily functions as an organ system that absorbs nutrients from food to meet the nutritional requirements of the body. However, it is being increasingly recognized that the functions of the gut are not restricted to food processing.

The interface between the gut and the external environment is significantly larger than that between the skin or the respiratory tract and the external environment. This large surface area 
is occupied by enormous numbers of gut microbiome and a complex immune system.

Gut homeostatic regulation is a complex process that involves a delicate equilibrium to limit inflammation despite close contact with enormous numbers of luminal commensal microflora. The major components contributing to gut homeostasis include the epithelium, gut microbiome, and immune cells. The epithelium consists of a layer of intestinal epithelial cells and forms a protective physical barrier against commensal microflora inhabiting the gut lumen. ${ }^{6}$ Complex microfloramicroflora and host-microflora interactions affect immune system, epithelial and barrier function, as well as the large metabolic capacity of the gut microbiome. Conversely, the immune, epithelial and barrier functions of the gut affect the aforementioned interactions. In addition to this complex mechanism, interactions between external environmental factors and host gut immunity and microbiome are instrumental in creating a unique gut environment and maintaining gut integrity in each individual. ${ }^{1}$ External environmental factors include geographical and ecological factors, diet, and drugs such as proton pump inhibitors or antibiotics. Host factors include age, race, breastfeeding, and combined disaeses affecting immunity. ${ }^{2}$ Diet is known to be the most important among the external environmental factors and has received the most attention.

Much research has focused on dietary influences on the gut environment and the interaction between diet and gut microbiome. To date, the most common and widely accepted concept is that dietary components alter the composition of the gut microbiome and consequently affect gut homeostasis. Dietary changes induce rapid fluctuations in the gut microbiome. Long-term dietary preferences determine the type of gut microbiome composition, that is, "enterotypes" defined by the variation in the levels of 1 of 3 genera: Bacteroides, Prevotella, and Ruminococcus. High protein and fat consumption are associated with abundant Bacteroides and carbohydrate-rich diets with the Prevotella enterotype. ${ }^{7.8}$

Depending on the individual's enterotypes, nutrients which comes the intestines may regulate the immuno-modulatory function of gut-resident immune cells. Thus, different byproducts are produced depending on specific nutrients. For example, it is known that long-term dietary patterns and the composition of gut microbiome vary widely between native Africans and African Americans, 2 genetically similar populations. Native Africans are at a low risk for colon cancer, whereas African Americans show the highest risk of colon cancer among the general population in the United States. ${ }^{9}$ If the microbial community context acquired in childhood is maintained healthy, even if bad bacteria enter the gut, host's metabolism, immunity, and disease status will be less affected by the strains. Similarly, the response to dietary intervention will depend on the context. ${ }^{10}$ However, the current available technology cannot accurately predict the individual's complex processes involved in such interaction.

Interestingly, the brain-gut microbiome axis is a biochemical signaling pathway that affects an individual's dietary behavior. Changes in gut environment cause brain-gut microbiome axis alterations via several feedback mechanisms to modify the host's eating behavior, resulting in cravings or dysphoria for certain nutrients. ${ }^{4}$

Research has shown that dietary influences on gut homeostasis are mediated via gut microbiome. The negative effects of dietary emulsifiers were not observed in germ-free mice, suggesting that the emulsifier-induced compositional and functional modulation of the gut microbiome plays a key role in the adverse effects caused by emulsifiers. ${ }^{11}$ However, failure of gut homeostasis is not always a consequence of gut dysbiosis. High fat diet can increase intestinal permeability and stimulate Toll-like receptors (TLRs) in germ-free mice or after prolonged antibiotic therapy. Free fatty acids directly activate inflammatory pathways and induce cathepsin B release from lysosomal instability in addition to activation of nuclear factor$\kappa B$. Palmitic acid activates interleukin (IL)- $1 \mathrm{~b}$ and IL-18 through a pathway involving TLR2 and the NALP3 inflammasome and directly increases intestinal permeability, resulting in systemic endotoxemia. ${ }^{4}$

Recent researches have shown that gut microbial metabolites including short-chain fatty acids (SCFAs), long-chain fatty acids, and tryptophan metabolites from non-digestible carbohydrates (dietary fibers) could benefit the host immune system and intestinal barrier function, thereby promoting gut homeostasis. SCFAs are primarily derived from bacterial fermentation not derived from dietary sources and serve as an energy source for host epithelial cells. SCFAs act as signaling molecules and possess anti-inflammatory, immunomodulatory, and anti-oxidative properties, and improve mucosal barrier function. ${ }^{3,4}$ Gut dysbiosis may compromise the metabolic activities of the gut microbiome and interfere with the generation of protective microbial metabolites. ${ }^{5}$

Therefore, a well-balanced healthy diet is essential for the development and maintenance of a healthy gut environment to ensure effective interactions between the host's immune 
system, epithelial barrier, and gut microbiome in a way that protects the host from disease.

\section{THE ASSOCIATION BETWEEN SPECIFIC DIETARY PATTERNS AND INTESTINAL DISEASES}

High species diversity is a key feature of the gut microbiome in healthy individuals. Specific external factors such as antibiotic usage, infection, and/or dietary changes can alter the composition of microbiome producing a non-homeostatic milieu. These changes are usually reversible in healthy individuals with marked diversity of gut microbiome. However, if the extraneous agents overpower the homeostatic capacity of the gut microbiome, severe disruption of the ecosystem can result in a reduction in microbiota diversity and resilience, with consequent tissue injury. ${ }^{12}$

In patients with IBD, the most prominent form of a diseased gut, a functional microbial dysbiosis was found by metagenomics studies including the European MetaHIT Project. ${ }^{13,14}$ Additionally, metabolomic analysis of breath or feces samples revealed reduced butyrate, acetate, and trimethylamine and elevated amino acid levels in patients with IBD. Deficient production of SCFAs is observed in patients with UC..$^{15,16}$

Accumulating evidences which suggest that diet is a significant etiopathogenetic contributor, or at least an aggravating factor in some intestinal diseases have been increasing and being accepted convincingly.

Researchers have reported an association between the consumption of red meat and the pathogenesis of intestinal disease. Colonic fermentation of large quantities of protein/red meat produces bacterial metabolites such as indoles, ammonia, and sulfides, which can harm the gut epithelium. ${ }^{17-19}$ Notably, constant exposure of the gut epithelium to such agents can contribute to colorectal cancer. ${ }^{20}$

Most ingested fat is digested and absorbed in the small intestine and does not reach the colon. However, bile salts excreted into the small intestine participate in the digestion of fats and undergo deconjugation and eventually reach the colon and affect the microbiome. Thus, dietary fats indirectly reduce the microbiota diversity and affect its composition, leading to impaired metabolism and inflammation. ${ }^{12,21}$ Another indirect effect of dietary fats is that they increase gut epithelial permeability and facilitate diffusion of lipopolysaccharides and other bacterial components across the gut epithelium, leading to chronic systemic inflammation. ${ }^{22}$ Adherent invasive Escherichia coli were shown to colonize an animal colitis model

\section{fed a Western $\operatorname{diet}^{23}$}

Emulsifiers are incorporated into most processed foods to enhance texture and stability. Recent studies have reported that the consumption of dietary emulsifiers disrupted hostmicrobiome interactions and promoted intestinal inflammation and colonic carcinogenesis. ${ }^{24}$

Dietary factors have received considerable attention as important etiopathogenetic contributors to IBD because the rapid increase in the incidence and prevalence of IBD can be attributed to dietary factors, at least to some extent. Genetic susceptibility alone in the available literature cannot explain the rapid upward curve of disease trends reported by epidemiological studies, just explaining a small fraction of disease development. ${ }^{14,20,25-27}$

The global increase in the incidence of IBD appears to be strongly associated with a Western lifestyle. Reportedly, various dietary components are known to negatively affect the composition of gut microbiome and/or cause or aggravate inflammation in animal models of IBD. These substances include high-fat/high-sugar diets, gluten, maltodextrin, emulsifiers, titanium dioxide nanoparticles, luminal iron, aluminum (a food chain contaminant), artificial sweeteners, and dietary phosphate, which are common constituents of the Western diet. ${ }^{11,24,28-32}$

Clinical studies of IBD have also investigated dietary components including cereals, fiber-containing foods, bread, sugar and sugar-containing foods, fruits and vegetables, fat, sucrose, starch, or total carbohydrate, and protein or energy drinks. ${ }^{14,3,34}$ While high-fat diets have been strongly implicated as an aggravating factor in animal models of CD but inconclusive in those of $U C,{ }^{14}$ a few clinical retrospective epidemiological studies have reported that the high-fat diet affects the clinical course of both, CD and UC. ${ }^{35,36}$ A significant association has been reported between high meat intake and the risk of UC relapse. ${ }^{33}$ The IBD study using the European Prospective Investigation into Cancer and Nutrition database showed no associations between total dietary carbohydrate, sugars (monosaccharides and disaccharides), or starch intakes and the odds of developing CD and UC. ${ }^{37}$ Animal studies have reported a potential association between food additives and IBD; however, the clinical significance of this finding remains unclear because most current investigations including those utilizing food questionnaires cannot completely and accurately evaluate the consumption of food additives that influence these conditions. Large-sized well-designed epidemiological studies are warranted to investigate this issue. ${ }^{14}$ Meanwhile, a prospective study has reported that consumption of large 
quantities of fiber, particularly fruits was associated with a lower risk of $\mathrm{CD}$, might being potential preventive components, although no such association was observed with respect to UC. ${ }^{38}$

Interestingly, it has been reported that the association between diet and IBD development may be stronger in patients with genetic predisposition and in those with gut dysbiosis. Studies have shown a gene-diet interaction affecting the risk of IBD in children who consumed a diet with a high omega 6/ omega 3 ratio in that these children were predisposed to $\mathrm{CD}$ if they were also carriers of specific gene variants for fatty acid metabolism. $^{39}$

With regard to the pathogenesis of celiac disease, it is well known that gluten activates zonulin, which increases intestinal epithelial permeability, leading to the phenomenon of "leaky gut, $^{\text {"30 }}$ which usually resolves following elimination of the offending (trigger) agent from the diet.

For the development and exacerbation of diverticulitis, red meat plays a key role via several potential mechanisms. It promotes chronic low-grade systemic inflammation and several components of red meat such as heme, N-nitroso compounds, and amines affect colonic epithelial homeostasis, as mentioned earlier. Prolonged intake of a diet rich in red meat can predispose to obesity, which is a known risk factor for diverticulitis. ${ }^{4-43}$

The increasing consumption of processed/unprocessed meat and a low-fiber diet is also associated with colorectal cancer, a malignancy typically associated with Western culture and lifestyles. Protein residues and fat-stimulated bile acids are also metabolized by the microbiome into inflammatory and/or carcinogenic metabolites, which increase the risk of neoplastic progression. ${ }^{17,44}$

For malnutrition, a significant association with gut microbiome has also been reported. Studies involving fecal transplants into gnotobiotic mice have observed that the malnutrition phenotype could be transferred with the microbiome from the Kwashiorkor twin. ${ }^{45}$ A comparative study between malnourished children in underdeveloped countries and healthy children in developed countries showed higher diversity of microbiome in the latter group. Low species diversity was associated with reduced metabolite production in the malnourished children, which can significantly affect development of the gut epithelium, leading to poor development of the GI system. Additionally, it may directly negatively affect absorption and production of certain essential amino acids, which are particularly important if the children get food that lacks these amino acids. In other words, altered bacterial inter- actions can cause reduced production of several essential amino acids in malnourished children. ${ }^{46}$ Thus, childhood malnutrition can affect maturation and development of organs secondary to disturbances in the nutrient uptake mechanism of a major nutrient.

\section{THE ROLE OF NUTRITIONAL THERAPY IN THE TREATMENT OF INTESTINAL DISEASE}

\section{Nutritional Therapy for Adequate Nutrient Supplementation}

Malnutrition is usually considered a medico-social issue only in a few underdeveloped countries and typically characterized by not having enough to eat (undernutrition).

However, individuals in developed countries are also at risk, particularly hospitalized patients. Notably, the definition of malnutrition has expanded to include deficiencies, excesses, or imbalances in an individual's energy and/or nutrient intake. In this article, we have restricted the definition of malnutrition to deficiencies.

Intestinal failure is the most representative gut condition in which nutritional therapy forms a major component of treatment. It occurs secondary to several inflammatory intestinal conditions such as CD or ischemic bowel disease. The range and type of nutritional therapy required in patients with intestinal failure depend on the extent and location of the diseased intestinal segments mainly in small intestine, which show disruption in absorptive capacity. If clinical presentations of the intestinal failure show a severe condition such as short bowel syndrome, intravenous supplementation for fluid, electrolyte and various nutrients would be a major component of the treatment strategy. Although limited evidence and guidelines are available regarding the optimal timing and methods of implementation and discontinuation of enteral nutrition (EN) or parenteral nutrition (PN), it is usually recommended that reintroduction of EN should be attempted as soon as patients recover from the severe intestinal disease. The insufficient energy not met by EN alone can be fulfilled by PN, which is called supplemental PN.

The multifactorial etiology of undernutrition in intestinal diseases includes lack of nutritional intake secondary to GI symptoms, malabsorption in the diseased segments of the gut, excessive GI losses secondary to diarrhea or fistula, and the influences of medications such as steroid and sulfasalazine. ${ }^{47}$ Other less common conditions affecting nutrition include missing teeth, orofacial pain conditions, salivary dysfunction, 
and oral complications during cancer treatment and certain GI disorders. These conditions are relatively unrelated to intestinal diseases themselves. ${ }^{48}$

Malabsorption is a major contributor to underweight in adults with CD during remission. ${ }^{49}$ Previous reports in the available literature had described that patients with IBD showed a higher basal metabolic rate than healthy controls, secondary to the action of inflammatory cytokines in the former. ${ }^{50}$ However, recent reports have shown that the energy requirements of patients with IBD were similar to those of the healthy population. Adults with IBD may show increased metabolic activity during episodes of severe disease associated with systemic disturbances (fever and tachycardia) compared to remission states; however, resting energy expenditure can be offset by reduction of physical activity. Changes in resting energy expenditure are not significant in mild disease states, even in children. ${ }^{47}$

Regardless of the diagnosis, accurate assessment of the patient's current nutritional status is important to fulfill the energy and protein requirements for optimal nutritional supplementation and to improve the prognosis of the disease. Correcting nutrient deficiencies and malnutrition in hospital and outpatient settings can reduce comorbidities, shorten recovery times, and reduce readmission rates. ${ }^{47,51}$

Knowledge regarding the sites and mechanism of absorption of key nutrients is essential to determine the appropriate micronutrients that should be administered to a patient with intestinal disease and to accurately predict deficiency states of micronutrients based on the intestinal segments affected by disease. For example, vitamin $B_{12}$ absorption occurs exclusively in the terminal ileum; therefore, vitamin $B_{12}$ supplementation is essential in patients undergoing terminal ileal resection or in those with severe inflammation of the terminal ileum because nutrient absorption is markedly affected in such cases.

\section{Dietary Intervention as a Therapeutic Approach}

Dietary intervention refers to any alteration or treatment in an individual's diet with a planned goal, which is usually designed to improve the individual's overall health. The 3 principal models of dietary intervention include supplementation with selected dietary components, exclusion of selected dietary components, or use of dietary formulas to replace a normal diet. ${ }^{52}$

Another aspect of dietary intervention is based on the understanding that diet significantly affects gut microbiome with consequent changes in the gut environment, as mentioned earlier. Metabolism of the host's immune and nonimmune cells, as well as that of the gut microbiome is altered in intesti- nal inflammation. Thus, the nutrient demands of the host and/ or the microbiome may be altered in a setting of GI disease. With regard to selecting treatment strategies, an accurate understanding of the appropriate dietary changes that could restore gut microbial diversity is important for optimal therapeutic dietary intervention.

Extensive research focusing on the effects of diet on the onset and exacerbation of GI disease has triggered interest in the utility of dietary modification as a treatment option. However, to date, few well-designed clinical trials and large-scale, randomized prospective studies have been performed to conclusively establish the optimal diet to treat specific diseases or induce/maintain remission. Meta-analyses reported in the literature have included small-scale studies.

Celiac disease is the most common disease for which dietary modification remains the main treatment. However, celiac disease is a chronic immune-mediated enteropathy triggered by dietary gluten, which serves as an antigen. Thus, it differs from the previously mentioned condition that involves complex interactions with the gut environment including microbiome. Currently, strict adherence to a gluten-free diet (GFD) is the only recommended treatment, which however is not useful in all patients. GFD has been shown to promote mucosal healing, reduce serum levels of celiac antibodies, improve nutritional deficiencies and bone health, and increase body fat. ${ }^{53}$

The efficacy of medical treatment is variable and the underlying pathophysiology remains largely unclear in patients with IBS, and the role of dietary interventions in patients with IBS is being widely investigated. FODMAP is an acronym for fermentable oligosaccharides, disaccharides, monosaccharides and polyols, which constitute poorly absorbed and rapidly fermented carbohydrates that are osmotically active and increase the small intestinal luminal water content, as well as intraluminal hydrogen and methane content secondary to colonic bacterial fermentation. Thus, FODMAPs can cause functional bowel symptoms including diarrhea, gas, abdominal pain, and flatulence in susceptible individuals. ${ }^{54}$ Several studies have shown that a low-FODMAP diet alleviates IBS symptoms. The adequate symptom relief was observed in $68 \%$ to $76 \%$ of patients with IBS who maintained the low-FODMAP diet over 4 to 8 weeks. ${ }^{55-58}$ However, the following concerns warrant consideration in this regard: (1) An interesting paradox observed in such cases is that a low-FODMAP diet markedly reduces the luminal concentration of Bifidobacteria in the gut. It is well known that gut dysbiosis observed in patients with IBS is char- 
acterized by a reduction in Bifidobacteria species, which might be associated with an unfavorable symptom profile. However, some FODMAPs function as prebiotics (e.g., fructo- and galacto-oligosaccharides) and presumably produce beneficial effects on the GI microbiome. A study has shown that shortterm FODMAP restriction correlated with reduced numbers of luminal Bifidobacterium spp. and Faecalibacterium prausnitzii. $^{54,56,59}$ (2) Long-term dietary restrictions can precipitate nutritional deficiencies because FODMAPs are constituents of a lot of food items. Therefore, a few researchers recommend a strategy comprising short-term FODMAP restriction for symptom control, followed by reintroduction of FODMAPs using food challenges to personal tolerance and finally, avoidance of only high FODMAP foods that trigger symptoms. ${ }^{14} \mathrm{~A}$ recent study showed that long-term FODMAP restriction was a nutritionally adequate and acceptable strategy in $>50 \%$ of the patients receiving dietary intervention. ${ }^{60}$

Studies have shown that FODMAPs could cause functional GI symptoms even in patients with IBD. . 14,61,62 $^{-10}$

Soluble fiber absorbs water and turns to gel during digestion and appears to improve IBS symptoms. Insoluble fiber increases stool volume, and soluble fiber is digested by colonic microbiome to form SCFAs, which nourish the colonic mucosal cells, improve mucus production, and may decrease inflammation at the cellular level. Although bran was not shown to be beneficial, there is no evidence to indicate any harmful effect associated with its use. A few studies have investigated the role of bran; however, most results are speculative and based on uncontrolled data. ${ }^{63,64}$

Dietary intervention, which aims to limit potential nutritional hazards, is an important treatment option in patients with IBD to induce and extend disease remission. ${ }^{14}$ Exclusive enteral nutrition (EEN) is an active nutritional intervention that serves as primary induction therapy for active pediatric CD. EEN is the most extensively researched dietary intervention for induction of remission in mild-to-moderate pediatric and adult $C D .{ }^{65}$ EEN was reported to induce clinical remission in approximately $80 \%$ of pediatric patients; ${ }^{66}$ however, the efficacy of EEN in adults is unclear because few studies have been performed in adults and these were of poor quality. The therapeutic effect of EEN is attributed to bowel rest, anti-inflammatory action, restoration of the epithelial barrier, favorable changes in the gut microbiome, and importantly, the exclusion of specific dietary components. ${ }^{67,68}$ Several researchers have reported that EEN decreased pro-inflammatory and increased anti-inflammatory molecules such as transforming growth factor $\beta{ }^{69,70}$ A mouse model of CD showed that EEN maintained normal gut barrier function and integrity with resolution of inflammation. ${ }^{71}$ To date, EEN-induced changes in gut microbiome remain inconclusive. Several studies have reported that EEN causes an extensive reduction in bacterial diversity, changes community-level metabolic functions and, at least initially, may increase microbial dysbiosis. With regard to taxonomic shifts, reportedly, EEN reduces the abundance of Firmicutes (including Faecalibacterium), Bacteroides/Prevotella, and Proteobacteriaceae and increases the abundance of Bacteroidetes. ${ }^{72-74}$ A metagenomic study reported that EEN increased genes that play a major role in cell growth and renewal and possibly in tissue healing, in spite of the similar change of microbiota with the previous unfavorable changes. ${ }^{72}$ Mechanisms of action of EEN warrant further research to gain a deeper understanding of the complex interplay between epigenetic, immunological, and microbiological changes.

Dietary intervention may also play a role in the prevention of diverticulitis, a common condition observed in clinical practice. It was previously presumed that nuts and food items with small seeds could lodge in the diverticula and cause inflammation. However, there is lack of scientific evidence to prove the role of nuts and seeds in the causation of diverticulitis flares. Data suggests that a high-fiber diet that includes insoluble fiber, such as wheat bran or fiber-based cereals reduces symptomatic disease or hospitalization for diverticular disease. ${ }^{75-77}$ Dietary fiber benefits patients with diverticular disease via the following mechanisms of action: (1) It increases stool bulk and decreases colonic pressures and stool transit time; (2) A high-fiber diet affects the composition and metabolism of gut microbiome and via the diet-gut microbiome interaction, it increases the production of SCFAs, which are essential for the maintenance of colonic epithelial integrity and may also reduce systemic inflammation; (3) An inverse correlation is observed between dietary fiber intake and obesity. ${ }^{41,78,79}$ In contrast, red meat intake, which might promote chronic low-grade inflammation, is reportedly strongly associated with symptomatic diverticular disease. ${ }^{80,81}$

A recent large cohort study reported that a "prudent diet" decreased and a Western diet increased the risk of diverticulitis. Alcohol or caffeine intake was not significantly associated with diverticular disease. Moreover, more recent or current dietary intake showed a stronger association than chronic or previous intake. ${ }^{41}$ The term "prudent diet" was introduced to describe the cholesterol-controlled diet in the Anti-Coronary Program of the New York City Department of Health since 
$19577^{82}$ The "prudent diet" is one that is rich in fruits, vegetables, whole grains, legumes, poultry, and fish. ${ }^{83}$

An analysis of the role of diet in diverticular disease must be based on the understanding that dietary patterns are closely associated with lifestyle and cultural behavior. For example, it is observed that men consuming the "prudent diet" usually tend to be more physically active and are less likely to be smokers or consume alcohol. In cases of incidental diverticulitis, non-dietary risk factors, such as genetics, obesity, physical activity, and NSAID use may be closely associated with the development of the disease. ${ }^{83}$

The micronutrient and polyphenol content of diet should also be considered because these constituents affect microbial ecology, including its composition and metabolic activities.

\section{Role of Probiotics/Prebiotics as Specific Microbial- Based Nutritional Therapy}

Probiotics and prebiotics are natural dietary components that improve the intestinal environment and are recently being used as biotherapeutic agents for microbial-based therapy, comparable to fecal microbiota transplantation. Various probiotics are commonly used to prevent dysbiosis in patients undergoing prolonged antibiotic or immunosuppressive therapies. The mechanisms of action of probiotics are dependent on microbial strains.

With regard to immunological functions, probiotic strains produce growth factors that strengthen the gut epithelium/gut barrier and antimicrobial substances such as SCFAs, bacteriocins, hydroperoxides, bile acids, and lactic acid, which destroy pathogens. ${ }^{84}$ Moreover, probiotics activate host immune responses via release of cellular components from the strains and improve macrophage and lymphocyte activity. ${ }^{85}$

With regard to non-immunological function, probiotics/prebiotics improve digestion and absorption, compete with potential pathogens for nutrients and intestinal adhesion sites, modulate $\mathrm{pH}$ alterations, and promote agglutination of pathogens and sequestration of metabolic toxins. ${ }^{86,87}$ These agents are also known to inhibit apoptosis and participate in mucin synthesis and tissue repair, thereby enhancing the protective function of the gut barrier. ${ }^{88}$

However, probiotics or prebiotics have shown limited efficacy in microbial modulation of active GI disease. ${ }^{87,89}$ Additionally, lack of standard therapeutic regimens, small-sized study samples, and poor disease characterization are limitations of the clinical and experimental studies that have investigated the efficacy of probiotics/prebiotics. Thus, their role remains inconclusive in this context. Nonetheless, probiotics are shown to be potentially useful in the management of IBS, diverticulitis (with some success), and IBD, in addition to a few other conditions. $^{59,90}$

Akkermansia muciniphila, an intestinal symbiont that plays a key role in mucin degradation, is recently gaining increasing attention as a promising probiotic secondary to its ability to improve host metabolic functions and immune responses. A few animal studies have reported that the ingestion of live $A$. muciniphila counteracts the metabolic disturbances caused by a high-fat diet including fat-mass gain, metabolic endotoxemia, adipose tissue inflammation, and insulin resistance and aids in restoration of normal mucus layer thickness by reducing endotoxemia. A. muciniphila could serve as a novel potential therapeutic agent in various GI diseases including colitis (particularly IBD), colon cancer, and other microbiota-related diseases. Preliminary data from human studies suggest that oral administration of A. muciniphila is safe; however, further studies are required to verify the effectiveness and safety of this agent. ${ }^{91-93}$

\section{NUTRITION RESEARCHES: GLOBAL TRENDS AND FUTURE PERSPECTIVES}

To date, much research has focused on gaining a deeper understanding of the genetic and the microbial association with regard to GI diseases. Studies have also been performed to develop effective and safe pharmacological agents for GI diseases; however, limited research data have been available with regard to the role of nutrition or diet in this context. Presently, $\mathrm{diet} /$ nutrition is increasingly recognized as one of the important factors in the pathogenesis and management of GI disease. Therefore, several developed countries are actively engaged in developing and commercializing newer forms of nutritional therapies, in addition to planning and coordination to accelerate the pace of research.

Although much is known to date regarding the association between nutrition/diet and gut health, several gaps remain in research and knowledge in this domain. The association between nutrition and gut health is extremely complex; therefore, multidisciplinary collaborative research is warranted in future. For example, modification of patients' long-term dietary habits requires psychosocial or sociocultural approaches in addition to scientific and medical strategies.

The National Institutes of Health (NIH) Nutrition Research Task Force has developed the Strategic Plan for NIH Nutrition 
Research since October 2016 to guide to accelerate basic, translational, and clinical research, as well as training for nutrition research over the next 10 years. This plan aims to improve health and prevent or treat nutrition/diet-induced conditions. ${ }^{48}$ With regard to IBD, the Dietitians' Committee of the European Crohn's and Colitis Organisation working group in collaboration with other experts in microbiology, physiology, and medicine reviewed the evidences investigating the role of diet and nutritional therapy in the development and management of IBD. ${ }^{14}$ These findings are expected to provide data for future researchers investigating the role of diet and nutrition in IBD.

Presently, the focus of research in the domain of gut microbiome is shifting towards the functional capacity of the microbiome in that "what they are doing" is being viewed as more important than "who is there." Compared with previous studies that investigate whether any individual components of gut microbiome are beneficial or pathogenic, current studies has increasingly recognized the gut microbiome as an ecosystem based on symbiotic relationships between members of the microbial communities and between these communities and the host. ${ }^{48}$ The use of "omic" (e.g., metabolomic, genomic, transcriptomic, and proteomics) approaches is a potential trend in short-term intervention studies and long-term observational studies to identify and validate potential biomarkers of dietary patterns, food groups, or food components of interest.

Notably, establishing causality between diet, microbiome, and diseases are still needed. Longitudinal studies are required to investigate early life exposure to factors including diet and other environmental agents affecting prevention and development of diseases, as well as to gain detailed knowledge regarding the microbiome.

Caloric restriction and the association between diet, energy metabolism, and biorhythm have received attention for the management of various systemic and metabolic diseases, including obesity, diabetes, hypertension, and stroke. However, their role in intestinal diseases remains unclear.

\section{CONCLUSION}

Nutritional therapy is not limited to being a mode of energy and protein supplementation or a strategy to prevent and/or manage disease-induced malnutrition. Dietary patterns significantly affect the gut microbiome, and diet-induced microbial alterations can affect the gut environment; therefore, nutritional therapy can be viewed as a cost-effective therapeutic and preventive strategy for intestinal health. Further research is warranted to gain a deeper understanding of the complex interplay between various dietary nutrients, host immunity, and the gut microbiome to improve the effectiveness of dietary interventions to treat intestinal diseases.

\section{FINANCIAL SUPPORT}

The author received no financial support for the research, authorship, and/or publication of this article.

\section{CONFLICT OF INTEREST}

No potential conflict of interest relevant to this article was reported.

\section{AUTHOR CONTRIBUTION}

Writing and approval of final manuscript: Kim SE.

\section{ORCID}

Kim SE https://orcid.org/0000-0002-6310-5366

\section{REFERENCES}

1. Wlodarska M, Kostic AD, Xavier RJ. An integrative view of microbiome-host interactions in inflammatory bowel diseases. Cell Host Microbe 2015;17:577-591.

2. Simrén M, Barbara G, Flint HJ, et al. Intestinal microbiota in functional bowel disorders: a Rome foundation report. Gut 2013;62:159-176.

3. Thorburn AN, Macia L, Mackay CR. Diet, metabolites, and "Western-lifestyle" inflammatory diseases. Immunity 2014;40: 833-842.

4. Tilg H, Moschen AR. Food, immunity, and the microbiome. Gastroenterology 2015;148:1107-1119.

5. Sugihara K, Morhardt TL, Kamada N. The role of dietary nutrients in inflammatory bowel disease. Front Immunol 2019;9: 3183.

6. Fournier BM, Parkos CA. The role of neutrophils during intestinal inflammation. Mucosal Immunol 2012;5:354-366.

7. Arumugam M, Raes J, Pelletier E, et al. Enterotypes of the human gut microbiome. Nature 2011;473:174-180.

8. Wu GD, Chen J, Hoffmann C, et al. Linking long-term dietary patterns with gut microbial enterotypes. Science 2011;334: $105-108$ 
9. Ou J, Carbonero F, Zoetendal EG, et al. Diet, microbiota, and microbial metabolites in colon cancer risk in rural Africans and African Americans. Am J Clin Nutr 2013;98:111-120.

10. Griffin NW, Ahern PP, Cheng J, et al. Prior dietary practices and connections to a human gut microbial metacommunity alter responses to diet interventions. Cell Host Microbe 2017; 21:84-96.

11. Chassaing B, Koren O, Goodrich JK, et al. Dietary emulsifiers impact the mouse gut microbiota promoting colitis and metabolic syndrome. Nature 2015;519:92-96.

12. Zoetendal EG, de Vos WM. Effect of diet on the intestinal microbiota and its activity. Curr Opin Gastroenterol 2014;30:189195.

13. Qin J, Li R, Raes J, et al. A human gut microbial gene catalogue established by metagenomic sequencing. Nature 2010;464:5965.

14. Sigall-Boneh R, Levine A, Lomer M, et al. Research gaps in diet and nutrition in inflammatory bowel disease: a topical review by D-ECCO Working Group [Dietitians of ECCO]. J Crohns Colitis 2017;11:1407-1419.

15. Bodelier AG, Smolinska A, Baranska A, et al. Volatile organic compounds in exhaled air as novel marker for disease activity in Crohn's disease: a metabolomic approach. Inflamm Bowel Dis 2015;21:1776-1785.

16. Le Gall G, Noor SO, Ridgway K, et al. Metabolomics of fecal extracts detects altered metabolic activity of gut microbiota in ulcerative colitis and irritable bowel syndrome. J Proteome Res 2011;10:4208-4218.

17. Yao CK, Muir JG, Gibson PR. Review article: insights into colonic protein fermentation, its modulation and potential health implications. Aliment Pharmacol Ther 2016;43:181-196.

18. Devkota S, Wang Y, Musch MW, et al. Dietary-fat-induced taurocholic acid promotes pathobiont expansion and colitis in IL10-/- mice. Nature 2012;487:104-108.

19. Gruber L, Kisling S, Lichti P, et al. High fat diet accelerates pathogenesis of murine Crohn's disease: like ileitis independently of obesity. PLoS One 2013;8:e71661.

20. Lee D, Albenberg L, Compher C, et al. Diet in the pathogenesis and treatment of inflammatory bowel diseases. Gastroenterology 2015;148:1087-1106.

21. Le Chatelier E, Nielsen T, Qin J, et al. Richness of human gut microbiome correlates with metabolic markers. Nature 2013; 500:541-546.

22. Moreira AP, Texeira TF, Ferreira AB, Peluzio Mdo C, Alfenas Rde C. Influence of a high-fat diet on gut microbiota, intestinal permeability and metabolic endotoxaemia. Br J Nutr 2012;
108:801-809.

23. Martinez-Medina M, Denizot J, Dreux N, et al. Western diet induces dysbiosis with increased E coli in CEABAC10 mice, alters host barrier function favouring AIEC colonisation. Gut 2014;63:116-124.

24. Viennois E, Merlin D, Gewirtz AT, Chassaing B. Dietary emulsifier-induced low-grade inflammation promotes colon carcinogenesis. Cancer Res 2017;77:27-40.

25. Jostins L, Ripke S, Weersma RK, et al. Host-microbe interactions have shaped the genetic architecture of inflammatory bowel disease. Nature 2012;491:119-124.

26. Molodecky NA, Soon IS, Rabi DM, et al. Increasing incidence and prevalence of the inflammatory bowel diseases with time, based on systematic review. Gastroenterology 2012;142:4654.

27. Loddo I, Romano C. Inflammatory bowel disease: genetics, epigenetics, and pathogenesis. Front Immunol 2015;6:551.

28. Nickerson KP, McDonald C. Crohn's disease-associated adherent-invasive Escherichia coli adhesion is enhanced by exposure to the ubiquitous dietary polysaccharide maltodextrin. PLoS One 2012;7:e52132.

29. Roberts CL, Rushworth SL, Richman E, Rhodes JM. Hypothesis: increased consumption of emulsifiers as an explanation for the rising incidence of Crohn's disease. J Crohns Colitis 2013;7:338-341.

30. Sugihara K, Masuda M, Nakao M, et al. Dietary phosphate exacerbates intestinal inflammation in experimental colitis. J Clin Biochem Nutr 2017;61:91-99.

31. Rodriguez-Palacios A, Harding A, Menghini P, et al. The artificial sweetener Splenda promotes gut proteobacteria, dysbiosis, and myeloperoxidase reactivity in Crohn's disease-like ileitis. Inflamm Bowel Dis 2018;24:1005-1020.

32. Suez J, Korem T, Zeevi D, et al. Artificial sweeteners induce glucose intolerance by altering the gut microbiota. Nature 2014;514:181-186.

33. Jowett SL, Seal CJ, Pearce MS, et al. Influence of dietary factors on the clinical course of ulcerative colitis: a prospective cohort study. Gut 2004;53:1479-1484

34. Sakamoto N, Kono S, Wakai K, et al. Dietary risk factors for inflammatory bowel disease: a multicenter case-control study in Japan. Inflamm Bowel Dis 2005;11:154-163.

35. Jantchou P, Morois S, Clavel-Chapelon F, Boutron-Ruault MC, Carbonnel F. Animal protein intake and risk of inflammatory bowel disease: the E3N prospective study. Am J Gastroenterol 2010;105:2195-2201.

36. Racine A, Carbonnel F, Chan SS, et al. Dietary patterns and 
risk of inflammatory bowel disease in Europe: results from the EPIC study. Inflamm Bowel Dis 2016;22:345-354.

37. Chan SS, Luben R, van Schaik F, et al. Carbohydrate intake in the etiology of Crohn's disease and ulcerative colitis. Inflamm Bowel Dis 2014;20:2013-2021.

38. Ananthakrishnan AN, Khalili H, Konijeti GG, et al. A prospective study of long-term intake of dietary fiber and risk of Crohn's disease and ulcerative colitis. Gastroenterology 2013;145:970977.

39. Costea I, Mack DR, Lemaitre RN, et al. Interactions between the dietary polyunsaturated fatty acid ratio and genetic factors determine susceptibility to pediatric Crohn's disease. Gastroenterology 2014;146:929-931.

40. Fasano A. Zonulin and its regulation of intestinal barrier function: the biological door to inflammation, autoimmunity, and cancer. Physiol Rev 2011;91:151-175.

41. Strate LL, Keeley BR, Cao Y, Wu K, Giovannucci EL, Chan AT. Western dietary pattern increases, and prudent dietary pattern decreases, risk of incident diverticulitis in a prospective cohort study. Gastroenterology 2017;152:1023-1030.

42. Rosemar A, Angerås U, Rosengren A. Body mass index and diverticular disease: a 28-year follow-up study in men. Dis Colon Rectum 2008;51:450-455.

43. Ley SH, Sun Q, Willett WC, et al. Associations between red meat intake and biomarkers of inflammation and glucose metabolism in women. Am J Clin Nutr 2014;99:352-360.

44. O'Keefe SJ. Diet, microorganisms and their metabolites, and colon cancer. Nat Rev Gastroenterol Hepatol 2016;13:691-706.

45. Smith MI, Yatsunenko T, Manary MJ, et al. Gut microbiomes of Malawian twin pairs discordant for kwashiorkor. Science 2013;339:548-554.

46. Kumar M, Ji B, Babaei P, et al. Gut microbiota dysbiosis is associated with malnutrition and reduced plasma amino acid levels: lessons from genome-scale metabolic modeling. Metab Eng 2018;49:128-142.

47. Forbes A, Escher J, Hébuterne X, et al. ESPEN guideline: clinical nutrition in inflammatory bowel disease. Clin Nutr 2017; 36:321-347.

48. National Institute of Diabetes and Digestive and Kidney Diseases. Draft Strategic Plan for NIH Nutrition Research. NIH: Bethesda, 2018.

49. Vaisman N, Dotan I, Halack A, Niv E. Malabsorption is a major contributor to underweight in Crohn's disease patients in remission. Nutrition 2006;22:855-859.

50. Capristo E, Mingrone G, Addolorato G, Greco AV, Gasbarrini G. Metabolic features of inflammatory bowel disease in a re- mission phase of the disease activity. J Intern Med 1998;243: 339-347.

51. Hebuterne X, Filippi J, Schneider SM. Nutrition in adult patients with inflammatory bowel disease. Curr Drug Targets 2014;15:1030-1038.

52. Lewis JD, Albenberg L, Lee D, Kratz M, Gottlieb K, Reinisch $\mathrm{W}$. The importance and challenges of dietary intervention trials for inflammatory bowel disease. Inflamm Bowel Dis 2017; 23:181-191.

53. Cichewicz AB, Mearns ES, Taylor A, et al. Diagnosis and treatment patterns in celiac disease. Dig Dis Sci 2019;64:2095-2106.

54. Staudacher HM, Irving PM, Lomer MC, Whelan K. Mechanisms and efficacy of dietary FODMAP restriction in IBS. Nat Rev Gastroenterol Hepatol 2014;11:256-266.

55. Halmos EP, Power VA, Shepherd SJ, Gibson PR, Muir JG. A diet low in FODMAPs reduces symptoms of irritable bowel syndrome. Gastroenterology 2014;146:67-75.

56. Staudacher HM, Lomer MC, Anderson JL, et al. Fermentable carbohydrate restriction reduces luminal bifidobacteria and gastrointestinal symptoms in patients with irritable bowel syndrome. J Nutr 2012;142:1510-1518.

57. Staudacher HM, Whelan K, Irving PM, Lomer MC. Comparison of symptom response following advice for a diet low in fermentable carbohydrates (FODMAPs) versus standard dietary advice in patients with irritable bowel syndrome. J Hum Nutr Diet 2011;24:487-495.

58. Böhn L, Störsrud S, Liljebo T, et al. Diet low in FODMAPs reduces symptoms of irritable bowel syndrome as well as traditional dietary advice: a randomized controlled trial. Gastroenterology 2015;149:1399-1407.

59. Staudacher HM, Whelan K. Altered gastrointestinal microbiota in irritable bowel syndrome and its modification by diet: probiotics, prebiotics and the low FODMAP diet. Proc Nutr Soc 2016;75:306-318.

60. O'Keeffe M, Jansen C, Martin L, et al. Long-term impact of the low-FODMAP diet on gastrointestinal symptoms, dietary intake, patient acceptability, and healthcare utilization in irritable bowel syndrome. Neurogastroenterol Motil 2018;30:e13154.

61. Prince AC, Myers CE, Joyce T, Irving P, Lomer M, Whelan K. Fermentable carbohydrate restriction (low FODMAP diet) in clinical practice improves functional gastrointestinal symptoms in patients with inflammatory bowel disease. Inflamm Bowel Dis 2016;22:1129-1136.

62. Gearry RB, Irving PM, Barrett JS, Nathan DM, Shepherd SJ, Gibson PR. Reduction of dietary poorly absorbed short-chain carbohydrates (FODMAPs) improves abdominal symptoms 
in patients with inflammatory bowel disease-a pilot study. J Crohns Colitis 2009;3:8-14.

63. Moayyedi P, Quigley EM, Lacy BE, et al. The effect of fiber supplementation on irritable bowel syndrome: a systematic review and meta-analysis. Am J Gastroenterol 2014;109:13671374.

64. Nagarajan N, Morden A, Bischof D, et al. The role of fiber supplementation in the treatment of irritable bowel syndrome: a systematic review and meta-analysis. Eur J Gastroenterol Hepatol 2015;27:1002-1010.

65. Rubio A, Pigneur B, Garnier-Lengliné H, et al. The efficacy of exclusive nutritional therapy in paediatric Crohn's disease, comparing fractionated oral vs. continuous enteral feeding. Aliment Pharmacol Ther 2011;33:1332-1339.

66. Grover Z, Muir R, Lewindon P. Exclusive enteral nutrition induces early clinical, mucosal and transmural remission in paediatric Crohn's disease. J Gastroenterol 2014;49:638-645.

67. Levine A. Exclusive enteral nutrition: clues to the pathogenesis of Crohn's disease. Nestle Nutr Inst Workshop Ser 2014;79: 131-140.

68. Sigall-Boneh R, Pfeffer-Gik T, Segal I, Zangen T, Boaz M, Levine A. Partial enteral nutrition with a Crohn's disease exclusion diet is effective for induction of remission in children and young adults with Crohn's disease. Inflamm Bowel Dis 2014;20:13531360.

69. de Jong NS, Leach ST, Day AS. Polymeric formula has direct anti-inflammatory effects on enterocytes in an in vitro model of intestinal inflammation. Dig Dis Sci 2007;52:2029-2036.

70. Meister D, Bode J, Shand A, Ghosh S. Anti-inflammatory effects of enteral diet components on Crohn's disease-affected tissues in vitro. Dig Liver Dis 2002;34:430-438.

71. Nahidi L, Leach ST, Mitchell HM, et al. Inflammatory bowel disease therapies and gut function in a colitis mouse model. Biomed Res Int 2013;2013:909613.

72. Quince C, Ijaz UZ, Loman N, et al. Extensive modulation of the fecal metagenome in children with Crohn's disease during exclusive enteral nutrition. Am J Gastroenterol 2015;110: 1718-1729.

73. MacLellan A, Moore-Connors J, Grant S, Cahill L, Langille MGI, Van Limbergen J. The impact of Exclusive Enteral Nutrition (EEN) on the gut microbiome in Crohn's disease: a review. Nutrients 2017;9:447.

74. Gerasimidis K, Bertz M, Hanske L, et al. Decline in presumptively protective gut bacterial species and metabolites are paradoxically associated with disease improvement in pediatric Crohn's disease during enteral nutrition. Inflamm Bowel Dis
2014;20:861-871

75. Aldoori WH, Giovannucci EL, Rockett HR, Sampson L, Rimm EB, Willett WC. A prospective study of dietary fiber types and symptomatic diverticular disease in men. J Nutr 1998;128:714719.

76. Crowe FL, Balkwill A, Cairns BJ, et al. Source of dietary fibre and diverticular disease incidence: a prospective study of UK women. Gut 2014;63:1450-1456.

77. Crowe FL, Appleby PN, Allen NE, Key TJ. Diet and risk of diverticular disease in Oxford cohort of European Prospective Investigation into Cancer and Nutrition (EPIC): prospective study of British vegetarians and non-vegetarians. BMJ 2011; 343:d4131.

78. Albenberg LG, Wu GD. Diet and the intestinal microbiome: associations, functions, and implications for health and disease. Gastroenterology 2014;146:1564-1572.

79. Qi L, van Dam RM, Liu S, Franz M, Mantzoros C, Hu FB. Wholegrain, bran, and cereal fiber intakes and markers of systemic inflammation in diabetic women. Diabetes Care 2006;29:207211.

80. Aldoori WH, Giovannucci EL, Rimm EB, Wing AL, Trichopoulos DV, Willett WC. A prospective study of diet and the risk of symptomatic diverticular disease in men. Am J Clin Nutr 1994;60:757-764.

81. Manousos O, Day NE, Tzonou A, et al. Diet and other factors in the aetiology of diverticulosis: an epidemiological study in Greece. Gut 1985;26:544-549.

82. Livingston GE. Proceedings: the prudent diet. What? Why? How? Prev Med 1973;2:321-328.

83. Stollman N. The importance of being (dietarily) prudent. Gastroenterology 2017;152:934-936.

84. Konieczna P, Akdis CA, Quigley EM, Shanahan F, O'Mahony L. Portrait of an immunoregulatory Bifidobacterium. Gut Microbes 2012;3:261-266.

85. Markowiak P, Śliżewska K. Effects of probiotics, prebiotics, and synbiotics on human health. Nutrients 2017;9:1021.

86. Gagliardi A, Totino V, Cacciotti F, et al. Rebuilding the gut microbiota ecosystem. Int J Environ Res Public Health 2018;15: 1679.

87. Basso PJ, Câmara NOS, Sales-Campos H. Microbial-based therapies in the treatment of inflammatory bowel disease: an overview of human studies. Front Pharmacol 2019;9:1571.

88. Caballero-Franco C, Keller K, De Simone C, Chadee K. The VSL\#3 probiotic formula induces mucin gene expression and secretion in colonic epithelial cells. Am J Physiol Gastrointest Liver Physiol 2007;292:G315-G322. 
89. Jonkers D, Penders J, Masclee A, Pierik M. Probiotics in the management of inflammatory bowel disease: a systematic review of intervention studies in adult patients. Drugs 2012;72: 803-823.

90. Derwa Y, Gracie DJ, Hamlin PJ, Ford AC. Systematic review with meta-analysis: the efficacy of probiotics in inflammatory bowel disease. Aliment Pharmacol Ther 2017;46:389-400.

91. Zhang T, Li Q, Cheng L, Buch H, Zhang F. Akkermansia muciniphila is a promising probiotic [published online ahead of print April 21, 2019]. Microb Biotechnol. https://doi.org/10.1111/ 1751-7915.13410.

92. Everard A, Belzer C, Geurts L, et al. Cross-talk between Akkermansia muciniphila and intestinal epithelium controls dietinduced obesity. Proc Natl Acad Sci U S A 2013;110:9066-9071.

93. Cani PD, de Vos WM. Next-generation beneficial microbes: the case of Akkermansia muciniphila. Front Microbiol 2017;8: 1765. 